\title{
Differential diagnosis of pituitary masses at magnetic resonance imaging
}

\author{
Salvatore Maria Corsello ${ }^{1} \cdot$ Rosa Maria Paragliola $^{1}$
}

Received: 28 December 2016 / Accepted: 6 January 2017 / Published online: 16 January 2017

(C) Springer Science+Business Media New York 2017

A recent paper published by Chaichana $\mathrm{K}$ et al. [1] describes an interesting case of pituitary abscess causing bitemporal headaches and mimicking lymphocytic hypophysitis at magnetic resonance imaging (MRI). The diagnosis had been made on the basis of the lack of clinical response to high-dose dexamethasone treatment, which led to a repeat MRI 3 months later and to eventually perform excisional biopsy which showed the presence of the pituitary abscess. The most interesting feature of this case is its unusual imaging characteristics, in conjunction with the clinical presentation, which caused it to be misdiagnosed as hypophysitis.

The use of MRI in the diagnosis of pituitary abscess has been first reported in 1989 [2] and the widespread use of MRI has improved the diagnostic sensitivity. Several case reports and case series have been reported, but the literature agrees on the difficulty in making the correct diagnosis prior to surgery, due to overlapping clinical, imaging and laboratory findings with other sellar lesions. In particular, being able to make a differential diagnosis with hypophysitis and with the more common pituitary adenomas is crucial, considering the different therapeutic approach.

Pituitary abscess represents a rare condition, which can occur "de novo" (60\% of cases) or after a pre-existing pituitary lesion [3]. Tumors may be more vulnerable to infections because of their impaired circulation, necrosis or local immunological impairment, albeit this cause-effect relationship is only speculative [4]. However, changes in

Salvatore Maria Corsello

corsello.sm@meridiaroma.it

1 Endocrinology Unit, Università Cattolica del Sacro Cuore, Largo Gemelli 8, Rome I-00168, Italy
MRI features of a patient with a pre-existing sellar tumor, especially after a known sinus infection, is highly suggestive for the development of pituitary abscess [5].

Since the first description, a pituitary abscess appears at MRI as a cystic lesion with iso-hypointense signal on T-1 weighted sequences and iso-hyperintense signal on T-2, suggestive for a liquefied lesion, even if the latter finding could also be consistent with the necrotic part of an adenoma or Rathke's cleft cyst [3]. After gadolinium administration, pituitary abscess demonstrates typical rim enhancement [3]. In particular, a recent case series shows this characteristic in about $67 \%$ of affected patients [6].

Other signs that may suggest the correct diagnosis are the disparity between the sphenoid findings (effusion within the sinus, wide sellar floor destruction) and the relatively small volume of the pituitary lesion [4]. Moreover, another important sign is the enhancement of the sellar lesion outline by MRI contrast, with simultaneous extension to the sphenoidal sinus [7]. Several papers also report that a pituitary abscess can appear as a high intensity area on diffusion-weighted imaging [8]. This technique, which provides valuable information on the diffusion properties of water molecules in tissues, can be used for the differential diagnosis between abscesses and other cystic lesions.

If present, all the above mentioned characteristics seem to be more specific of a pituitary abscess and can aid in the differential diagnosis.

Pituitary adenomas are the most common sellar tumors. In some cases, their slow growth can result in progressive enlargement of the sella, with possible extension into the suprasellar cistern, cavernous or sphenoidal sinuses, or dorsum sella. MRI typically reveals hypointense or isointense signal on precontrast $\mathrm{T} 1$-weighted sequences and isointense signal with the brain cortex on T2-weighted 
sequences. On post-contrast images, these lesions have a decreased enhancement compared with pituitary gland. In presence of cystic component, the lesion may show variable signal intensity because of the presence of proteins or blood [9].

Some of these radiological features can be present also in the hypophysitis. In this condition, the pituitary enhancement is usually intense, homogeneous, and symmetrical while the involvement of sella turcica floor or clivus or cavernous sinuses is rare [9].

However, cases of uncommon presentation of hypophysitis at MRI have been reported: a case of inhomogeneous enhancement (both before and after gadolidium), absence of the typical "tent-shape" appearance and asymmetrical extrasellar expansion with infiltration of the left cavernous sinus has been described [10].

In conclusion, even if MRI provides some distinct features for various pituitary lesions, the differential diagnosis may be challenging, due to overlapping imaging characteristics among different conditions or to atypical MRI presentations within each condition. Furthermore, clinical and laboratory findings may also be misleading. For such reasons, pituitary abscess, in spite of its rarity, should be included in the differential diagnosis of patients with headache or signs of pituitary dysfunction as well as in patients with pituitary mass and signs of meningeal inflammation.

\section{Compliance with ethical standards}

Conflict of interest The authors declare that they have no conflict of interests.

Ethical approval This article does not contain any studies with human participants or animals performed by any of the authors.

\section{References}

1. K. Chaichana, T. Larman, R. Salvatori, Pituitary abscess with unusual MRI appearance. Endocrine 54, 837-838 (2016)

2. M. Dickob, T. Scharphuis, P. Distelmaier, G. Hoffman, Diagnosis of hypophyseal abscess using MRI and high resolution CT. Neurochirurgia (Stuttg). 32, 184-186 (1989)

3. F. Liu, G. Li, Y. Yao, Y. Yang, W. Ma, Y. Li, G. Chen, R. Wang, Diagnosis and management of pituitary abscess: experiences from 33 cases. Clin. Endocrinol 74, 79-88 (2011)

4. R.A. Hanel, A. Koerbel, D.M. Prevedello, M.S. Moro, J.C. Araújo, Primary pituitary abscess: case report. Arq. Neuropsiquiatr. 60, 861-865 (2002)

5. S.N. Kroppenstedt, T. Liebig, W. Mueller, K.J. Gräf, W.R. Lanksch, A.W. Unterberg, Secondary abscess formation in pituitary adenoma after tooth extraction. J. Neurosurg. 94, 335-338 (2001)

6. L. Gao, X. Guo, R. Tian, Q. Wang, M. Feng, X. Bao, K. Deng, Y. Yao, W. Lian, R. Wang, B. Xing: Pituitary abscess: clinical manifestations, diagnosis and treatment of 66 cases from a large pituitary center over 23 years. Pituitary. (2016) [Epub ahead of print Sep 30]

7. D. Bossard, A. Himed, C. Badet, V. Lapras, R. Mornex, G. Fisher, T. Tavernier, M. Bochu, MRI and CT in a case of pituitary abscess. J. Neuroradiol. 19, 139-144 (1992)

8. T. Takayasu, F. Yamasaki, A. Tominaga, T. Hidaka, K. Arita, K. Kurisu, A pituitary abscess showing high signal intensity on diffusion-weighted imaging. Neurosurg. Rev. 29, 246-248 (2006)

9. J.L. Go, A.G. Rajamohan, Imaging of the sella and parasellar Region. Radiol. Clin. North. Am. 55, 83-101 (2017)

10. L. Curtò, M.L. Torre, O.R. Cotta, M. Losa, M.R. Terreni, L. Santarpia, F. Trimarchi, S. Cannavò, Lymphocytic hypophysitis: differential diagnosis and effects of high-dose pulse steroids, followed by azathioprine, on the pituitary mass and endocrine abnormalities-report of a case and literature review. Sci. World J. 10, 126-134 (2010) 